\title{
Scar Revision: Surgical and Nonsurgical Options
}

\author{
Sameep Kadakia, MD ${ }^{1}$ Yadranko Ducic, MD, FRCS(C), FACS² Ameya Jategaonkar, MD ${ }^{1}$ David Chan, MD 3 \\ ${ }^{1}$ Department of Facial Plastic Surgery, New York Eye and Ear Infirmary \\ of Mount Sinai, New York, New York \\ 2 Department of Facial Plastics, Otolaryngology and Facial Plastic \\ Surgery Associates, Fort Worth, Texas \\ Address for correspondence Yadranko Ducic, MD, FRCS(C), FACS, \\ Department of Facial Plastics, Otolaryngology and Facial Plastic \\ Surgery Associates, 923 Pennsylvania Avenue, Suite 100, Fort Worth, \\ TX 76104 (e-mail: yducic@sbcglobal.net).
}

3 Department of Otolaryngology, Indiana University School of

Medicine, Indianapolis, Indiana

Facial Plast Surg 2017;33:621-626.

\begin{abstract}
Keywords

- scar revision

- topical

- surgical

Facial scarring represents a challenging issue for the facial plastic surgeon owing to the unpredictable and variable nature of scar formation. The psychologic, cosmetic, and functional implications of facial scars oftentimes necessitate revision. It is important for the facial plastic surgeon to be aware of the invasive and noninvasive options to best educate patients in terms of treatment modalities. As there are multiple options available with no single option touted as superior, management of expectations and patient motivation will allow for realistic treatment planning with the greatest change of success.
\end{abstract}

The process of scar formation and wound healing (-Fig. 1) represents a variable and unpredictable course of the natural healing process. Although scar formation is crucial to the normal healing mechanism, facial scars can often become unsightly and cause patients to develop psychosocial insecurities given the degree of cosmetic deformity. Aside from the cosmetic deformation, scars on the face can have functional consequences as well depending on the degree and vector of contracture. The challenges and lack of unified treatment modalities have led to the evolution of multiple revision techniques, both surgical and nonsurgical. Although the literature evaluating efficacy of these modalities is broad and there exist many inconsistencies in outcomes, it is important for the facial plastic surgeon to understand the different options available for treatment.

Prior to undertaking any treatment, a full examination of the scar and a thorough understanding of the patient's dissatisfaction are important in formulating a patient-centric goal for improvement. As no single approach is applicable to all patients, it is paramount to discuss expectations with the patient, as many times complete resolution of the scar may not be possible and reorientation or camouflaging techniques may be feasible alternatives. A majority of reviews in the literature have focused on surgical or nonsurgical options for scar management without providing the reader with a single source for an overview of all options. In this article, we seek not to provide an in-depth discussion of every single modality for scar revision, but to provide the reader with a reference list that can then be explored further at the surgeon's discretion.

\section{Options for Scar Revision}

\section{Topical and Intralesional Options}

Topical creams are often the first-line treatment for scars due to their low cost, favorable side-effect profile, and ease of availability. Similarly, intralesional pharmacotherapies are commonly employed in the early treatment of scars. Topical creams such as vitamin E, antibiotic ointments, sunscreens, and petroleum jellies are often most effective in the acute/ immediate postoperative setting. These therapies work to optimize healing and minimize visible scarring. Nevertheless, there are some ointment/creams that are known to be effective in the treatment of certain scars. Intralesional therapies involve injections into the targeted scars and usually multiple injections over an extended time period. Often various intralesional therapies may be combined to improve the likelihood of successful conservative therapies.

\section{Silicone Products}

Silicone gels and sheeting have been shown to be effective in the treatment of cutaneous scars. Silicone-based products have become a mainstay in clinical practice and are known to reduce hypertrophy through sealing and hydrating dense scar tissue. ${ }^{1}$ In fact, silicone products are regarded as one of
Issue Theme Aesthetic Management of Upper and Midface Trauma; Guest Editor, Yadranko Ducic, MD, FRCS(C), FACS
Copyright (C) 2017 by Thieme Medical Publishers, Inc., 333 Seventh Avenue, New York, NY 10001, USA. Tel: +1(212) 584-4662.
DOI https://doi.org/ 10.1055/s-0037-1607446. ISSN $0736-6825$. 

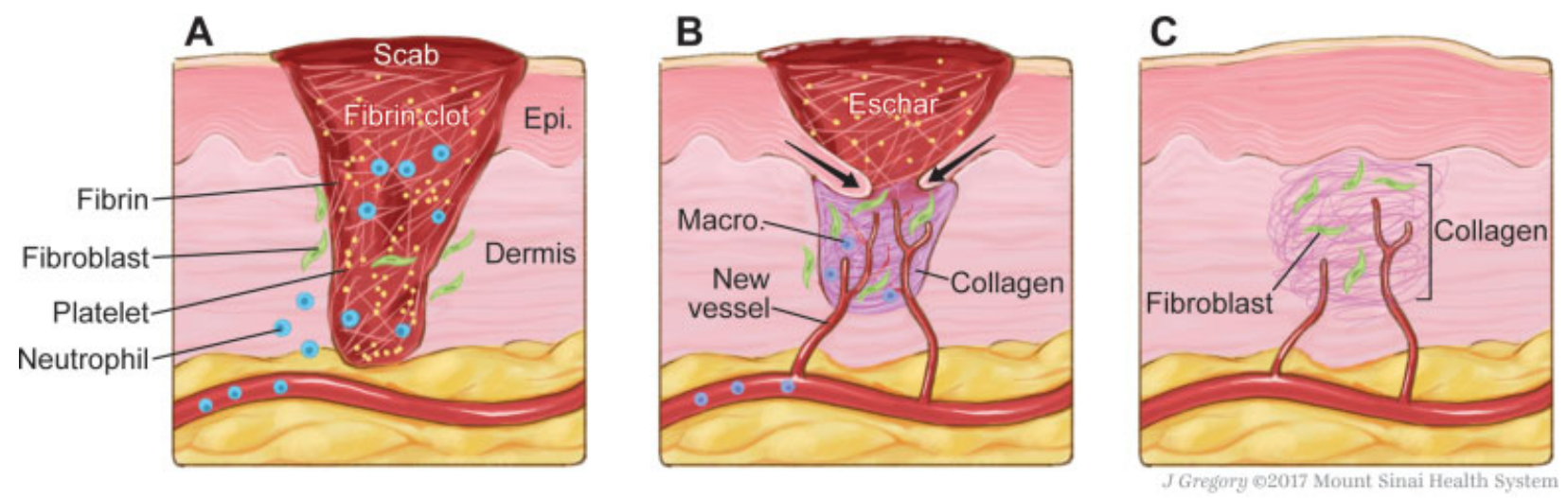

Fig. 1 Stages of wound healing. (A) Inflammatory phase. (B) Proliferative phase. (C) Remodeling phase.

the few options for scar therapy with sufficient backing by scientific evidence and considered by some to be the gold standard for noninvasive scar management techniques. ${ }^{2,3}$ Silicone sheeting typically consists of a sheet made from silicone gel reinforced by a silicone and/or polytetrafluoroethylene membrane. ${ }^{4}$

The majority of studies have looked at silicone gel sheeting; however, studies evaluating silicone-based gels have shown that these newer gels have efficacy that is comparable to sheeting, especially for facial scars. ${ }^{1,5,6}$ Silicone sheeting, which can be used to prevent or treat existing scars, can be used as long as the patient continues to obtain benefit, as the side effects are negligible. ${ }^{3}$ It is important to note that this modality should not be used on open wounds. A meta-analysis demonstrated that silicone sheeting significantly reduced the formation of hypertrophic scars in high-risk patients. ${ }^{7}$ Silicone-based products may be used in conjunction with intralesional injections of steroids or 5 -fluorouracil (5-FU).

\section{Imiquimod}

Imiquimod is an immune system modulator that functions by activating toll-like receptors(TLRs) and subsequently stimulating the production of cytokines and antigen presentation. Ultimately, this results in an increased immune response in treated tissues. Often used in the treatment of anogenital warts and as a topical therapy for basal cell carcinomas, imiquimod is also frequently used in the treatment of hypertrophic scars and keloids. ${ }^{8,9}$ It can be used alone; however, it is most commonly used after surgical or shave excision of the lesion, with daily application of $5 \%$ imiquimod cream to the scar. Most studies report frequent recurrences of keloids despite imiquimod. However, there is evidence to suggest that keloids of the pinna are particularly responsive to imiquimod. ${ }^{8,9}$

\section{Corticosteroids}

Corticosteroids have been a mainstay in the treatment of hypertrophic scars and keloids for decades. The vast preponderance of evidence for steroids has been with intralesional therapy. However, ointments/creams can be used as the firstline treatment prior to injections. Corticosteroids have been shown to reduce fibroblast proliferation and collagen synthesis. Steroids also suppress the production of inflammatory cytokines and alter gene expression in keratinocytes. This includes suppression of transforming growth factor-beta (TGF- $\beta$ ), which has been linked to the development of keloids. ${ }^{10}$

Intralesional corticosteroids remain a prime for keloids and second line for hypertrophic scars. ${ }^{5,11}$ Treatment usually consists of monthly intralesional injections with regular follow-up and reassessment to monitor the treatments. It is also important to note that steroid therapy can be paired with other treatments. Corticosteroids have also been shown to be effective as an adjunctive agent to other therapies including cryotherapy or 5-FU. ${ }^{6,12}$

\section{Bleomycin}

Bleomycin is a cytotoxic polypeptide that induces DNA strand breaks. Bleomycin functions by inhibiting the proliferation of fibroblasts and other inflammatory cells. Bleomycin has been shown to be effective in the treatment of cutaneous scars and keloids. Intralesional bleomycin injections have been shown to reduce pain/pruritus and result in scar flattening. Bleomycin can be combined with a local anesthetic to improve bleomycin uptake into cells. Bleomycin treatments usually involve single injections; however, the treatment can be repeated 3 to 4 weeks after the initial injection. It is also worth noting that bleomycin may be used alone or in conjunction with intralesional triamcinolone. ${ }^{5,6,12,13}$

\section{5-Fluorouracil}

5-fluorouracil is a pyrimidine analog that is cytotoxic due to its inhibition of thymidylate synthase. When injected into scars or applied topically, it inhibits the proliferation of fibroblasts. Clinically, it has been successfully used in the treatment of scars for decades and with excellent response rates. ${ }^{5} 5$-FU can be injected alone as monotherapy, or it can be combined with corticosteroids (usually triamcinolone). In a recent review of the literature, Shah et al report that keloid response rates with 5-FU alone range from 45 to $78 \%$. However, when combined with triamcinolone, response rates improved to $96 \%{ }^{14} 5$-FU can also be used in conjunction with laser therapies; however, 5-FU alone may achieve results comparable to those achieved in conjunction with the laser.

\section{Mitomycin C}

Mitomycin C is another cytotoxic agent that has been used to treat hypertrophic scars and keloids. Mitomycin $\mathrm{C}$ has been 
shown to be effective when used topically after surgical excision of keloids; however, its efficacy as a topical agent on unoperated tissue is yet to be demonstrated in the literature. ${ }^{5}$

\section{Dermabrasion}

Dermabrasion is a skin resurfacing technique that was first described in the 1930s. It was initially used for tattoo removal, but it has since proven itself effective in treating a variety of dermatologic conditions. Dermabrasion relies on a rotating abrasive tip (wire or diamond tip) to cause a controlled ablation of superficial skin layers. The goal is to remove layers until the level of the papillary-reticular dermal junction. Dermabrasion relies on proper technique to minimize thermal injury and to ensure appropriate depth of abrasion. The appropriate depth can be identified by pinpoint bleeding that marks the papillary dermis layer followed by the parallel collagen segment that marks the correct depth of abrasion. ${ }^{15,16}$

Subsequent healing is driven by the surrounding epithelium and results in a smoother scar without excess thickness. Dermabrasion has been shown to be effective in the treatment of a variety of scars including acne, surgical scars, actinic keratosis, rhinophyma, and facial rhytids. ${ }^{16-19}$ Ultimately, dermabrasion represents a simple and cost-effective option for the treatment of a variety of scars.

\section{Laser Therapies}

Various lasers have been used in a multitude of different manners to treat scars. Historically, lasers have been used either as ablative therapy or nonablative therapy. Photodynamic therapy (PDT) represents a newer technology that utilizes lasers for the treatment of a variety of skin conditions.

\section{Ablative Lasers}

Ablative lasers utilize the laser to ablate skin layers to the depth of the papillary dermis. $\mathrm{CO}_{2}$ lasers were first described for skin resurfacing in 1985. Subsequently, the erbium-doped yttrium aluminium garnet (Er:YAG) laser was introduced and today both are used for laser ablation. The $\mathrm{CO}_{2}$ laser creates a zone of thermal injury $200 \mu \mathrm{m}$ in depth, whereas the pulsed Er:YAG laser creates a zone of injury of approximately $50 \mu \mathrm{m}$. As such, the Er:YAG laser produces less thermal injury and has a lower depth of penetration. Hence, the Er:YAG laser may be titrated better than the $\mathrm{CO}_{2}$ laser. Both lasers are effective and can be used in the treatment of scars, skin rejuvenation, and other skin conditions including rhinophyma and the cutaneous manifestations of tuberous sclerosis. ${ }^{20}$

Fractional ablation is a newer technique that was first described in 2004 by Manstein et al as fractional photolysis. ${ }^{21}$ In fractional ablation, the laser is used to ablate tissue in the target field in a pixelated fashion. This leaves behind islands of healthy tissue that lead to faster healing and with fewer side effects. Tidwell et al recently published a double-blinded split scar study that showed that fractionated Er:YAG laser was superior to the fully ablative laser for scar revision. ${ }^{22}$

Ablative lasers do have their side effects and postprocedure erythema can last for weeks. Patients with darker skin are more likely to have pigmentary changes, ranging from postinflammatory hyperpigmentation to delayed hypopigmentation. Patients may also experience acneiform eruptions and worsening of scar from ablative laser treatments. ${ }^{23}$ Fractionated laser treatments represent a newer technique that may ameliorate some of these adverse effects.

\section{Nonablative Lasers}

Nonablative lasers represent a treatment modality for less severe lesions in patients who are seeking milder results and faster recoveries. Nonablative lasers aim to protect the epidermis and selectively destroy dermal tissues. Often it is the microvasculature within the dermis that is targeted by these lasers. Cooling of the epidermis with cryospray or air cooling can be used to protect the dermis from the effects of the laser. $^{20,23}$ Nonablative lasers use different gain media than lasers used in ablative laser treatments. The most commonly used nonablative lasers are the neodymium-doped yttrium aluminium garnet laser, pulsed potassium titanyl phosphate lasers, or diode lasers. These treatments are effective in mild to moderate wrinkles and similar lesions. However, these are not typically used for scar revision. ${ }^{20,23}$

\section{Photodynamic Therapy}

Photodynamic therapy utilizes an optically active drug that is delivered to targeted tissues and then activated by light. The first and most commonly used photosensitive agent is 5-aminolevulinic acid. Methyl aminolevulinic acid is another photoactive agent. Upon activation with light, reactive oxygen species are formed in the targeted tissue resulting in tissue ablation. This treatment methodology has been explored in a variety of medical/surgical disciplines and remains an active area of research. The literature contains reports of PDT being used to treat a variety of dermatologic conditions ranging from vascular malformations, to cancers, to scars. Some basic science work has been done showing the efficacy of PDT in killing keloid fibroblasts. ${ }^{24}$ Recently, there have been reports in the literature of PDT being used in the treatment of keloids and surgical scars. ${ }^{25}$ Most treatment protocols described in the literature involve weekly PDT sessions.

PDT represents a promising treatment modality for a variety of conditions. Treatment protocols will likely continue to evolve as more research is conducted on the efficacy of PDT and into novel photosensitizing agents. ${ }^{26,27}$

\section{Surgical Management}

Surgical management of scars represents an option for scar revision that can be undertaken primarily or following failure of conservative measures depending on the patient's motivation and goals. As scar revision oftentimes entails the "trading" of one scar for another, or simply the reorientation of a scar into a less conspicuous location, precision and planning are of utmost important. As patients have differing propensities to form scars, handling soft tissue in an atraumatic fashion while being aware of facial subunits and relaxed skin tension lines is crucial for a successful outcome. ${ }^{28}$ Multiple options exist for planning scar revision, with varying levels of complexity. Selected techniques along with descriptions and illustrations where appropriate are described below. 


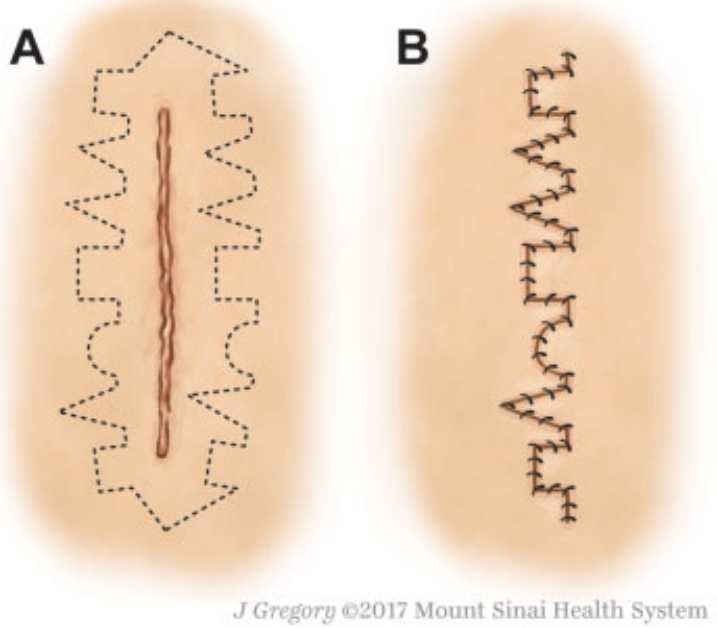

Fig. 2 Geometric broken line closure. (A) Initial scar with incision marking. (B) Final closure following excision of previous scar.

\section{Ellipse}

The simplest method of revising a scar is to excise an ellipse of tissue around it followed by primary closure of the wound edges. Estimating approximately 30-degree angles at the corners of the ellipse, the scar can be excised while placing the new incision line either within or parallel to a relaxed skin tension line. ${ }^{28}$ It is important to note that a multilevel closure with careful attention to redundant tissue at the edges is crucial to a cosmetically favorable outcome.

\section{Geometric Broken Line Closure}

For longer scars, the geometric broken line technique can be useful in camouflaging the scar as it creates an irregular appearance. Created with irregular and asymmetric geometric shapes, the scar is less perceptible to the human eye following closure (-Fig. 2 ).

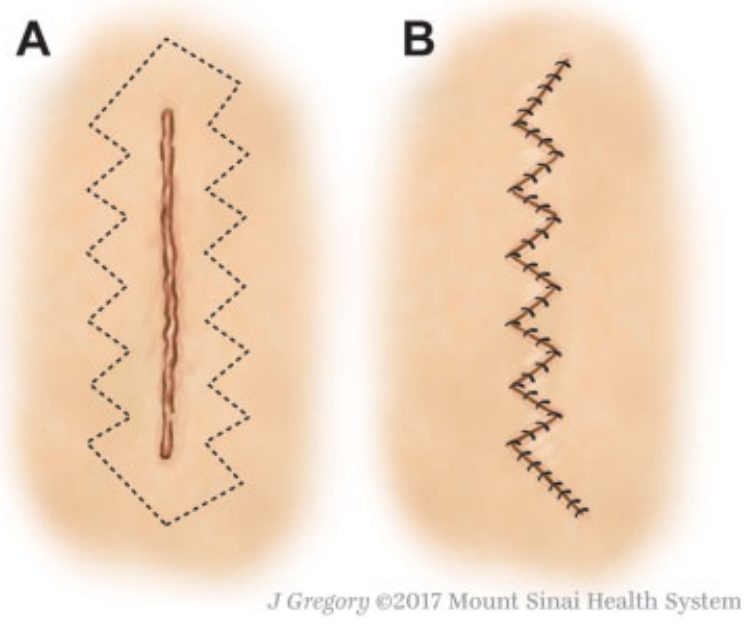

Fig. 4 W-plasty. (A) Initial scar with incision marked in irregular fashion. (B) Final closure following excision of previous scar.

\section{M-Plasty}

M-plasty is a technique of scar revision that is based on modifying an elliptical excision. In certain locations, healthy tissue may not want to be sacrificed and as such, the M-plasty saves more native tissue while maintaining similar angles as utilized in an ellipse technique (-Fig. $\mathbf{3}$ ).

\section{W-Plasty}

Another useful technique in camouflaging a scar that may not be in a favorable location, similar to the geometric broken line design, the $\mathrm{W}$-plasty also makes a scar more irregular and hence, less perceptible to the eye. The incision is designed by making consecutive "W" designs on both sides of the scar, followed by excision and reapproximation to give a jagged, saw-toothed appearance to the $\operatorname{scar}$ (-Fig. 4).
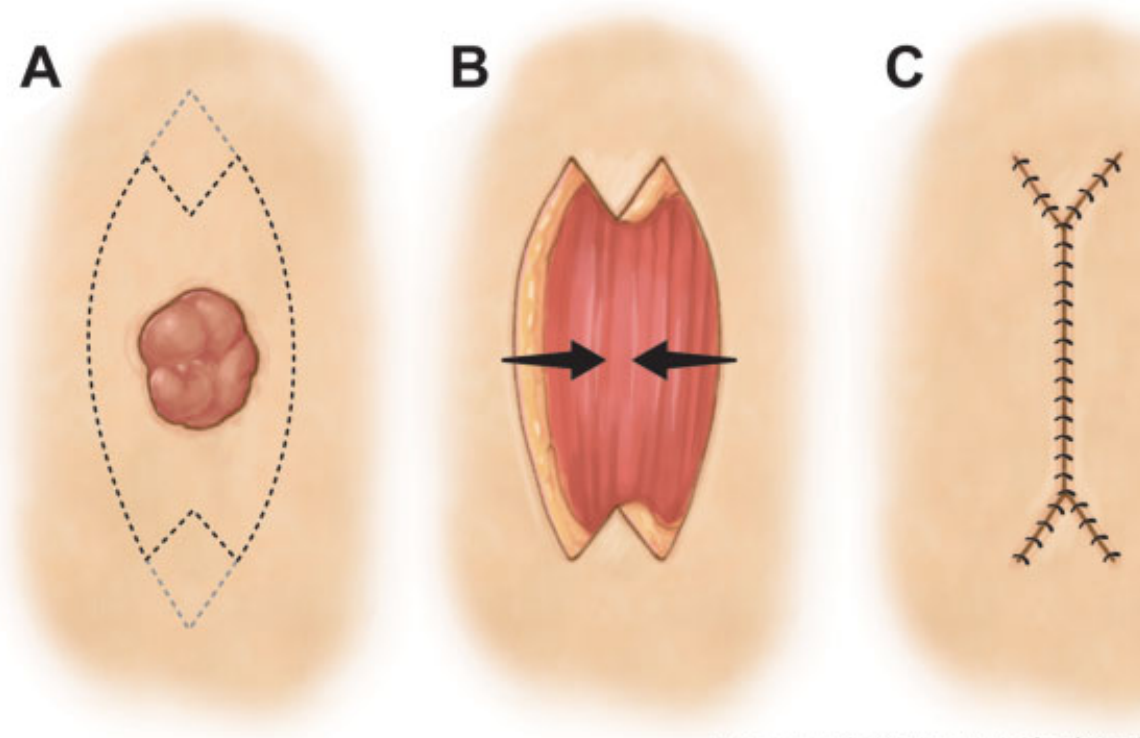

Fig. 3 M-plasty. (A) Initial defect with planned M-plasty incision. (B) Excised skin with vectors of movement. (C) Final closure of M-plasty. 

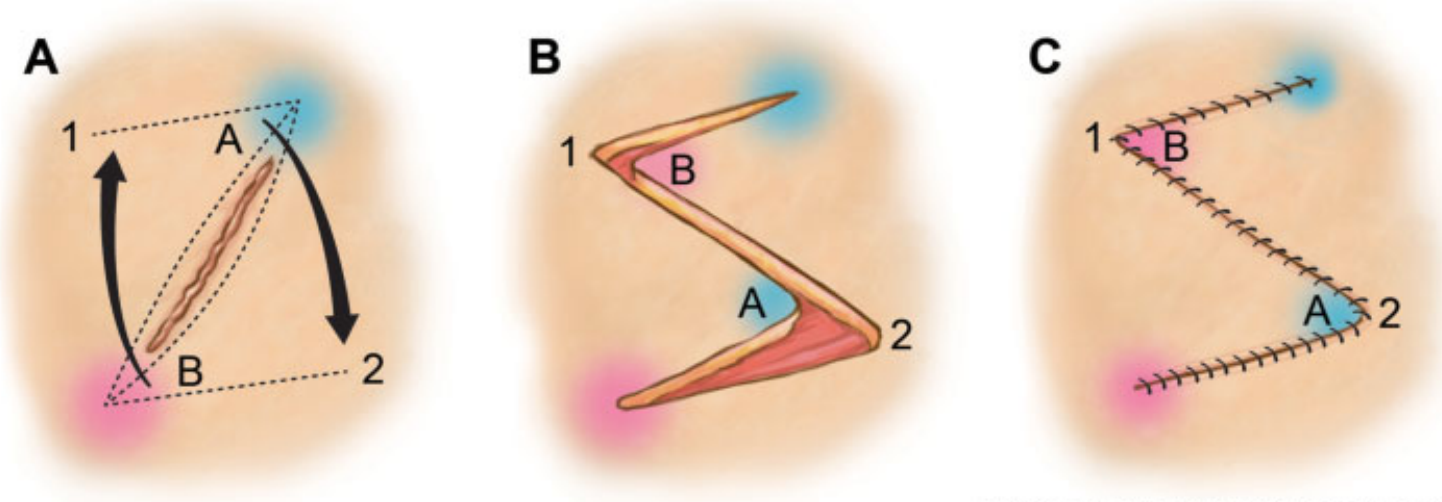

J Gregory @2017 Mount Sinai Health System

Fig. 5 Z-plasty. (A) Initial scar shown with incision markings and vectors of movement. (B) Fully incised "Z" with A and B representing congruent angles. (C) Closed incision showing reoriented and elongated scar.

\section{Z-Plasty}

Z-plasty is a versatile technique in scar revision used to lengthen and reorient the angles of a scar. The initial scar is used as the central portion of the " $Z$ " while obliquely oriented limbs are made on either side to rotate and change scar appearance. Typically, 30-degree angles lengthen a scar by $25 \%, 45$-degree angles lengthen by $50 \%$, and 60 -degree angles lengthen by $75 \%$ (-Fig. 5).

\section{Radiation Therapy}

Radiation therapy for scar management, particularly keloids, has been well described in the literature; however, the application of this therapy is highly dependent on institutions and practice patterns. It is the senior author's (Y.D.) belief that in some circumstances, single-dose same day postoperative external beam radiation for recurrent keloids can be a useful treatment adjunct. Song et al have reported that their experience of delivering a single-fraction electron beam radiation dose of $10 \mathrm{~Gy}$ immediately postoperatively led to no recurrence and no adverse side effects. ${ }^{29}$ Another 2016 studied found that the delivery of $18 \mathrm{~Gy}$ divided into three fractions within the first 36 hours postoperatively resulted in a $94 \%$ local control rate of intractable keloids, with no procedure-related complications. ${ }^{30} \mathrm{~A}$ recent metaanalysis in the plastic surgery literature analyzing 9,048 keloids treated with postoperative radiotherapy found that there was indeed a lower recurrence rate in patients treated postoperatively compared with those treated with radiotherapy alone. Moreover, they reported the lower recurrence rates with brachytherapy compared with electron beam and $\mathrm{X}$-ray. ${ }^{31}$ It appears that the use of postoperative radiation therapy is immensely helpful; however, the modality and fractionation regimen are yet to be agreed upon.

\section{Conclusion}

Facial scars can result in an unacceptable cosmetic appearance. Multiple options for the management of scars are available, including topic medications, intralesional injections, laser therapy, dermabrasion, photodynamic treatment, and surgical management. Although no single modality is superior, it is important for the practitioner to recognize the multifaceted approach to scar management keeping in mind the patient's goals and preferences. Management of expectations and clear communication can allow the patient to participate in their care and lead to greater satisfaction with posttreatment outcomes.

\section{Conflict of Interest}

None.

\section{References}

1 Mustoe TA. Evolution of silicone therapy and mechanism of action in scar management. Aesthetic Plast Surg 2008;32(01):82-92

2 Mustoe TA, Cooter RD, Gold MH, et al; International Advisory Panel on Scar Management. International clinical recommendations on scar management. Plast Reconstr Surg 2002;110(02):560-571

3 Monstrey S, Middelkoop E, Vranckx JJ, et al. Updated scar management practical guidelines: non-invasive and invasive measures. J Plast Reconstr Aesthet Surg 2014;67(08):1017-1025

4 Carney SA, Cason CG, Gowar JP, et al. Cica-Care gel sheeting in the management of hypertrophic scarring. Burns 1994;20(02):163-167

5 Gold MH, Berman B, Clementoni MT, Gauglitz GG, Nahai F, Murcia C. Updated international clinical recommendations on scar management: part 1-evaluating the evidence. Dermatol Surg 2014;40 (08):817-824

6 Gold MH, McGuire M, Mustoe TA, et al; International Advisory Panel on Scar Management. Updated international clinical recommendations on scar management: part 2-algorithms for scar prevention and treatment. Dermatol Surg 2014;40(08):825-831

7 O'Brien L, Pandit A. Silicon gel sheeting for preventing and treating hypertrophic and keloid scars. Cochrane Database Syst Rev 2006;(01):CD003826

8 Chuangsuwanich A, Gunjittisomram S. The efficacy of 5\% imiquimod cream in the prevention of recurrence of excised keloids. J Med Assoc Thai 2007;90(07):1363-1367

9 Viera MH, Vivas AC, Berman B. Update on keloid management: clinical and basic science advances. Adv Wound Care (New Rochelle) 2012;1(05):200-206

10 Xia W, Phan TT, Lim IJ, Longaker MT, Yang GP. Complex epithelialmesenchymal interactions modulate transforming growth factorbeta expression in keloid-derived cells. Wound Repair Regen 2004;12(05):546-556 
11 Hayashi T, Furukawa H, Oyama A, et al. A new uniform protocol of combined corticosteroid injections and ointment application reduces recurrence rates after surgical keloid/hypertrophic scar excision. Dermatol Surg 2012;38(06):893-897

12 Fang QQ Chen CY, Zhang MX, et al. The effectiveness of topical anti-scarring agents and a novel combined process on cutaneous scar management. Curr Pharm Des 2016;23(15):2268-2275

13 Saray Y, Güleç AT. Treatment of keloids and hypertrophic scars with dermojet injections of bleomycin: a preliminary study. Int J Dermatol 2005;44(09):777-784

14 Shah VV, Aldahan AS, Mlacker S, Alsaidan M, Samarkandy S, Nouri K. 5-Fluorouracil in the treatment of keloids and hypertrophic scars: a comprehensive review of the literature. Dermatol Ther (Heidelb) 2016;6(02):169-183

15 Lighthall JG, Fedok FG. Treating scars of the chin and perioral region. Facial Plast Surg Clin North Am 2017;25(01):55-71

16 Agarwal J, Kadakia S, Saman M. Current concepts in dermabrasion therapy. Am J Cosmet Surg 2016;33(03):140-144

17 Gold MH. Dermabrasion in dermatology. Am J Clin Dermatol 2003;4(07):467-471

18 Yarborough JM Jr. Ablation of facial scars by programmed dermabrasion. J Dermatol Surg Oncol 1988;14(03):292-294

19 Brenner MJ, Perro CA. Recontouring, resurfacing, and scar revision in skin cancer reconstruction. Facial Plast Surg Clin North Am 2009;17(03):469-487.e3

20 Wu EC, Wong BJ. Lasers and optical technologies in facial plastic surgery. Arch Facial Plast Surg 2008;10(06):381-390

21 Manstein D, Herron GS, Sink RK, Tanner H, Anderson RR. Fractional photothermolysis: a new concept for cutaneous remodeling using microscopic patterns of thermal injury. Lasers Surg Med 2004;34(05):426-438

22 Tidwell WJ, Owen CE, Kulp-Shorten C, Maity A, McCall M, Brown TS. Fractionated Er:YAG laser versus fully ablative Er:YAG laser for scar revision: results of a split scar, double blinded, prospective trial. Lasers Surg Med 2016;48(09):837-843

23 Brauer JA, Patel U, Hale EK. Laser skin resurfacing, chemical peels, and other cutaneous treatments of the brow and upper lid. Clin Plast Surg 2013;40(01):91-99

24 Chiu LL, Sun CH, Yeh AT, et al. Photodynamic therapy on keloid fibroblasts in tissue-engineered keratinocyte-fibroblast co-culture. Lasers Surg Med 2005;37(03):231-244

25 Ud-Din S, Thomas G, Morris J, Bayat A. Photodynamic therapy: an innovative approach to the treatment of keloid disease evaluated using subjective and objective non-invasive tools. Arch Dermatol Res 2013;305(03):205-214

26 Sakamoto FH, Izikson L, Tannous Z, Zurakowski D, Anderson RR. Surgical scar remodelling after photodynamic therapy using aminolaevulinic acid or its methyl ester: a retrospective, blinded study of patients with field cancerization. Br J Dermatol 2012; 166(02):413-416

27 Mamalis AD, Lev-Tov H, Nguyen DH, Jagdeo JR. Laser and lightbased treatment of keloids-a review. J Eur Acad Dermatol Venereol 2014;28(06):689-699

28 Watson D, Reuther MS. Scar revision techniques-pearls and pitfalls. Facial Plast Surg 2012;28(05):487-491

29 Song C, Wu HG, Chang H, Kim IH, Ha SW. Adjuvant single-fraction radiotherapy is safe and effective for intractable keloids. J Radiat Res (Tokyo) 2014;55(05):912-916

30 Jiang P, Baumann R, Dunst J, et al. Perioperative interstitial highdose-rate brachytherapy for the treatment of recurrent keloids: feasibility and early results. Int J Radiat Oncol Biol Phys 2016; 94(03):532-536

31 Mankowski P, Kanevsky J, Tomlinson J, Dyachenko A, Luc M. Optimizing radiotherapy for keloids: a meta-analysis systematic review comparing recurrence rates between different radiation modalities. Ann Plast Surg 2017;78(04):403-411 